\title{
Temporal brightness enhancement studied with a large sample of observers: Evidence for individual differences in brightness perception
}

\author{
RICHARD W. BOWEN and KATHRYN A. MARKELL \\ Loyola University of Chicago, Chicago, Illinois 60626
}

\begin{abstract}
Brightness vs. duration relations were measured for 80 naive observers using a method in which a short pulse (10-500 msec) was compared to a pulse $500 \mathrm{msec}$ longer in duration at the same luminance. Pulses were presented under two conditions of pulse asynchrony: simultaneous onset and simultaneous offset. Three classes of observers were found: Type A observers $(57 \%$ of all observers) showed temporal brightness enhancement (the Broca-Sulzer effect) for both simultaneous onset and offset conditions. Type B observers $133 \%$ of the sample) showed brightness enhancement for simultaneous offset but not onset. Type C observers (10\% of the sample) did not exhibit brightness enchancement under either asynchrony condition. The distribution of these classes was not affected by the specificity of instructions concerning the brightness judgment or by the sequencing (ordered vs. random) of stimuli. We analyze how these classes of observers might be explained either on the basis of differing neurophysiological mechanisms or responses or on the basis of differing psychophysical criteria. Further, we present a model of the data which illustrates how behaviorally distinct types of observers could be generated by continuous distributions within the general population of either perceptual criteria or neurophysiological responses.
\end{abstract}

The brightness of a pulse of light depends upon its exposure time. For pulses less than $50-100 \mathrm{msec}$, brightness increases with duration; for pulses greater than about $200 \mathrm{msec}$, brightness is constant with increasing pulse duration. Pulses of intermediate duration may appear significantly brighter than all shorter or longer equal-luminance stimuli, a phenomenon called "temporal brightness enchancement" or "the Broca-Sulzer effect" after Broca and Sulzer (1902).

It has been shown that the occurrence of the Broca-Sulzer effect is sensitive to the stimulus conditions selected for study (e.g., Bowen \& Nissen, 1979; Raab \& Osman, 1962; Wasserman, 1966). But it is generally assumed, as for most psychophysical phenomena, that if the Broca-Sulzer effect is seen by a given observer (usually an experienced observer) under a given set of stimulus conditions, it should likewise be seen by all possible observers.

However, we have frequently, but informally, observed that not all individuals will see the enhancement effect under identical stimulus conditions. Visitors to our laboratory are often asked to parti-

Supported by National Science Foundation Grant BNS 78-17779 to R. W. Bowen. We are grateful to Frank L. Slaymaker for advice concerning the statistical treatment of the data. We thank Vivianne C. Smith, Joel Pokorny, and Donald Hood for a critical reading of the manuscript. Two anonymous reviewers provided several useful suggestions and criticisms. cipate in our demonstration of the Broca-Sulzer effect. The demonstration consists of comparing a 100 -msec light pulse of moderate luminance $(100$ $1,000 \mathrm{~cd} / \mathrm{m}^{2}$ ) presented to one eye with a longer pulse (500 msec or more) of the same luminance presented to the other eye. In our demonstration, these pulses coterminate. The visitor says which pulse appeared brighter. Most observers indicate that the 100 -msec pulse seems definitely brighter (the BrocaSulzer effect). Some fraction of our visitors, however, claim that the longer pulse is brighter; coaxing or coaching on our part does not alter their assessment. In the past, we have been slightly embarrassed by such a visitor, but in view of the research to be reported here, we now indicate to the visitor that they are one of several fundamental types of observers of brightness.

In other words, our informal observations led us to postulate that in the general population there may exist different classes of observers based upon whether or not they manifest the Broca-Sulzer effect. As will be seen, we now suggest that there are actually three behavioral classes of observers, since we have observed that the occurrence or nonoccurrence of the effect can depend upon the temporal asynchrony between the long and short pulses to be compared.

In the present study, we measured brightnessduration relations for a large sample $(\mathrm{N}=80)$ of naive observers. ${ }^{1}$ The existence of different classes 
of observers might easily go unnoticed in a conventional psychophysical experiment, which seldom employs more than two to four observers.

Given that observers differ, we were also interested in three other parameters: (1) Could the responses of observers be influenced by the instructional set for the experiment? In particular, we wondered whether relatively detailed instructions which urged the observer to ignore differences in stimulus duration in assessing brightness might produce, for example, relatively more observers who showed the effect than instructions which merely requested the observer to make brightness judgments. (2) Would the order of comparisons affect the distribution of observers? Would, for example, a sequence of trials in which the shorter pulse was initially very brief and was increased in duration progressively over trials make the brightness enhancement effect more "obvious" to observers than a random trial sequence? (3) Does the Broca-Sulzer effect depend on a particular temporal asynchrony between pulses viewed simultaneously? According to Raab and Osman (1962), brightness enchancement (measured by a brightness matching task using a test and comparison pulse) occurs only when the pulses coterminate, and not when the pulses are presented with simultaneous onset or when the shorter pulse is presented during the exposure time of the longer pulse. However, the stimulus conditions employed by Raab and Osman, namely, spatially adjacent stimuli viewed binocularly, would tend to optimize lateral interactions between stimuli (Bowen \& Pokorny, 1978), and other investigators have shown that no effects of the temporal relation between stimuli are observed if care is taken to prevent mutual spatial influences between stimuli (Alpern, 1963; Boynton, 1961; Katz, 1964). We therefore thought it important to investigate the variable of temporal asynchrony.

\section{METHOD}

Brigtness-duration relations have been measured using brightness matching methods; a typical procedure has been to have the observer set a brightness match between test stimuli of various durations and a long-duration comparison stimulus of adjustable luminance. A schematic representation of possible results of such a procedure is given in Figure 1a. A function of this sort is taken to reflect differences in brightness among equal-luminance pulses of differing duration.

In the present study, we employed an alternative method which we developed (Bowen \& Nissen, 1979; Bowen \& Pokorny, 1978). On each experimental trial, the observer views two equal-luminance pulses of different durations, presented haploscopically to the left and right eyes, and is asked to report which pulse ("left" or "right") appeared brighter. These pulses may have either simultaneous onset or simultaneous offset. From trial to trial, the position of the longer and shorter pulses is varied. The data are in the form of functions relating the percentage of trials on which the longer pulse is judged brighter to the duration of the shorter pulse.

Figures $1 \mathrm{~b}$ and $\mathrm{lc}$ illustrate schematically two possible outcomes of such a procedure. If, as in Figure 1a, the Broca-Sulzer effect is present under a given set of stimulus conditions, the data will (a)

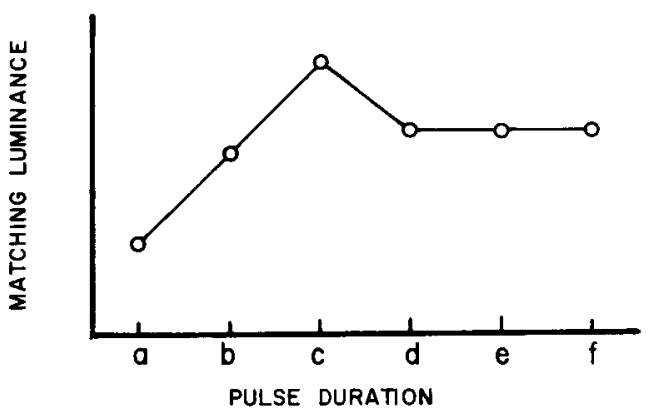

(b)

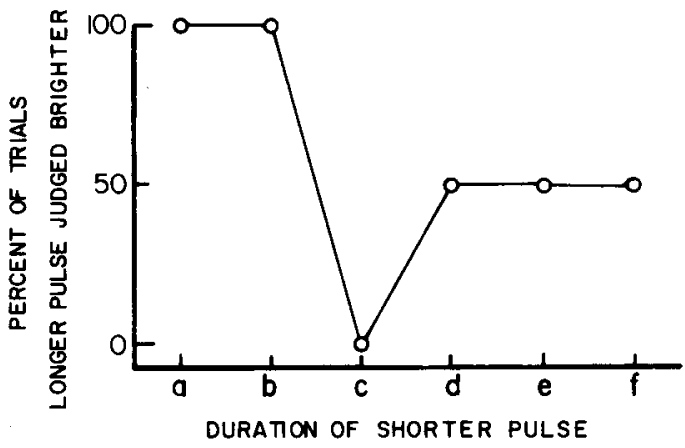

(c)

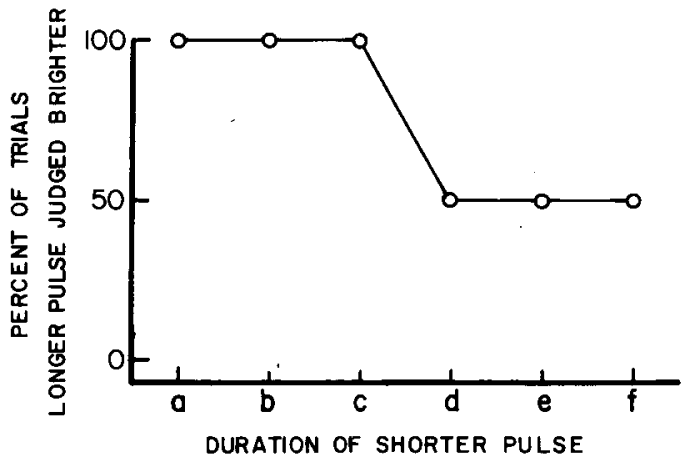

Figure 1.(a) Schematic representation of the results of a brightness matching procedure for studying the Broca-Sulzer effect. (B) Hypothetical results to be expected with the present method if temporal brightness enchancement occurs (see text). (c) Hypothetical outcome for the nonoccurrence of brightness enchancement using our method.

have a pattern similar to that in Figure 1b. For short pulses, where brightness increases with duration, the longer of two pulses should be judged brighter on $100 \%$ of all trials. For long pulses, where brightness is independent of duration, the longer pulse should be judged brighter on $50 \%$ of all trials, since no basis for a brightness discrimination exists. But when intermediateduration pulses are compared to longer pulses, the longer pulse should be judged brighter on $0 \%$ of all trials, since brightness is at a maximum at intermediate values of pulse duration.

On the other hand, if the Broca-Sulzer effect does not occur, the data should ideally exhibit a transition from $100 \%$ to $50 \%$ with no values below $50 \%$, as shown in Figure 1c.

We have emphasized previously (Bowen \& Nissen, 1979; Bowen \& Pokorny, 1978) that, while this method does not estimate the absolute level of brightness, it is very sensitive to the occurrence of brightness enhancement.

\section{Observers}

Eighty college students ( 36 males, 44 females) participated in the experiment to fulfill an introductory psychology course 

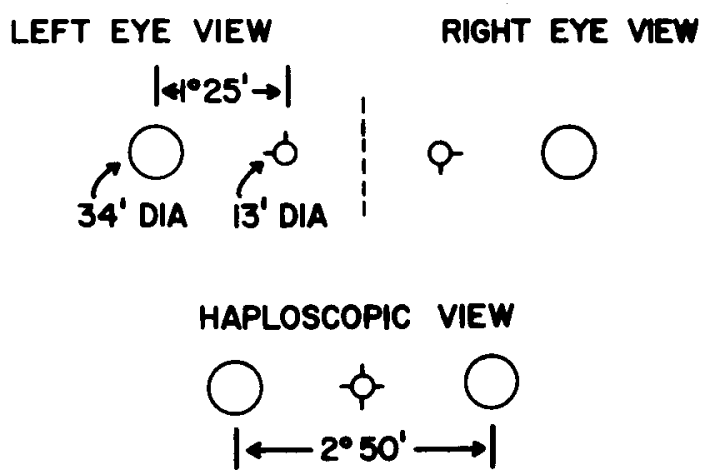

Figure 2. The stimulus array.

requirement. All observers were screened for Snellen visual acuity and had visual acuity of at least $6 / 6$ (with or without corrective lenses) in both eyes.

\begin{abstract}
Apparatus
The apparatus (Bowen \& Pokorny, 1978) consisted of a threechannel haploscopic optical projection system, which generated the achromatic stimulus array on rear-projection screens (Figure 2). In haploscopic view, the fixation targets in each eye were fused by the observer, and the pulse target locations ( $34 \mathrm{~min}$ in diameter) were separated by $2 \mathrm{deg} 50 \mathrm{~min}$. The fixation targets had a luminance of $20 \mathrm{~cd} / \mathrm{m}^{2}$, and the pulsed targets had a luminance of $100 \mathrm{~cd} / \mathrm{m}^{2}$. Luminance measurements were made regularly, using an Ilford photometer calibrated against a Gamma Model 220 standard lamp. The stimulus array was viewed at a distance of $.4 \mathrm{~m}$ with the observer's head positioned by a chinrest.
\end{abstract}

\section{Procedure}

The experiment was conducted by one of the authors (K. A. M.). At the beginning of the experimental session, the observer was shown how to fuse binocularly the circular portions of the fixation targets by adjusting the interocular distance between the stimuli. Minor adjustments in target luminance provided an exact brightness match for each observer. Finally, the observer was given a set of instructions (see below). Observers were assigned at random to one of the four experimental conditions discussed below.

In the course of a single 1-h experimental session, the observer made relative brightness judgments on a total of 320 pulse pairs. On half of these trials, the pulses had simultaneous onset, and on the other half, they had simultaneous offset. There were eight shorter pulse durations for the shorter pulse: $10,32,50$, $63,80,100,200$, and $500 \mathrm{msec}$. The longer pulse had a duration equal to that of the shorter pulse duration plus $500 \mathrm{msec}$.

Stimuli were presented in blocks of five trials at a given shorter pulse duration. Within each block, the position of the shorter pulse, right or left, was varied randomly. Under each asynchrony condition (simultaneous onset vs. simultaneous offset), each of the eight shorter pulse-duration pairs was presented to the subject 20 times, 10 times with the shorter pulse to the left, and 10 times with the shorter pulse to the right. The intertrial interval was approximately $5 \mathrm{sec}$.

\section{Experimental Conditions}

The four conditions consisted of:

(1) General instructions, ordered sequence-The observers were given the following instructions: "In this experiment, we would like you to make some judgments of apparent brightness. On each trial, you will see two flashes of light. You should respond by saying which of the two flashes, left or right, was brighter. If the left-hand flash seemed brighter, say 'left,' if the right-hand flash seemed brighter, say "right' " Blocks of five trials were presented to the observers in this condition in an ascending order, from a shorter pulse duration of $10 \mathrm{msec}$ to a shorter pulse duration of $500 \mathrm{msec}$, all at a given pulse asynchrony. Replications for asynchrony conditions were counterbalanced according to either an ABBA pattern or a BAAB pattern $(A=$ simultaneous onset, $\mathrm{B}=$ simultaneous off set).

(2) General instructions, random sequence-Observers under this condition were given the general instructions, but trial blocks for both asynchrony conditions were presented in a random order for four complete replications, yielding a total of 20 trials for each pulse-pair/asynchrony condition.

(3) Specific instructions, ordered sequence-These observers were given the general instructions supplemented by: "The flashes you will see will differ in how long they are presented. One flash will be short, one will be long. Please ignore these differences in time, and pay attention only to the apparent brightness. If a certain flash, short or long, seems to be definitely brighter at any instant during the presentation, you should indicate so by calling that one brighter. Again, ignore any differences between the flashes except for their brightness." Trial blocks were presented in an ascending order, as in the first condition.

(4) Specific instructions, random sequence-The observers were given the specific instructions, and trial blocks were presented to them in a random order.

\section{RESULTS}

The brightness-duration relation is reflected in a plot of the percent of trials on which the longer pulse in a comparison was judged brighter as a function of the duration of the shorter pulse. Figures 3-6 each show data for the four major experimental conditions as labeled on the figures, for simultaneous onset (open circles, dashed lines) and simultaneous offset (filled circles, solid lines). Within each figure, we have arranged the data into three groups of observers, designated A, B, and C. Observers are categorized on the basis of whether or not they show brightness enhancement for each condition of pulse asynchrony. We based our empirical criterion for the occurrence of the Broca-Sulzer effect on the properties of the binomial distribution. In particular, with 20 trials for each data point and an assumed probability of responding "longer pulse brighter" of .5, 6 trials or less out of $20(30 \%)$ is significantly lower than $50 \%$ in a binomial distribution. (The probability of 6 or less judgments of "longer judged brighter" is less than .057.)

For Type A observers, brightness enhancement effects were obtained for both simultaneous onset and offset conditions, where the brightness enhancement effect is indicated by any values of "longer pulse judged brighter" that are significantly lower than $50 \%$. Some observers in this class (for example, $5,22,43$, and 68 ) have relatively high values of "longer pulse judged brighter" for comparisons with relatively brief pulses and values near $50 \%$ for comparisons with longer pulses, a pattern resembling the "ideal" results presented in Figure 1b. For other observers, the functions are lower overall than the ideal case. Across the Type A observers, the func- 
GENERAL INSTRUC./ORDERED SEQ.
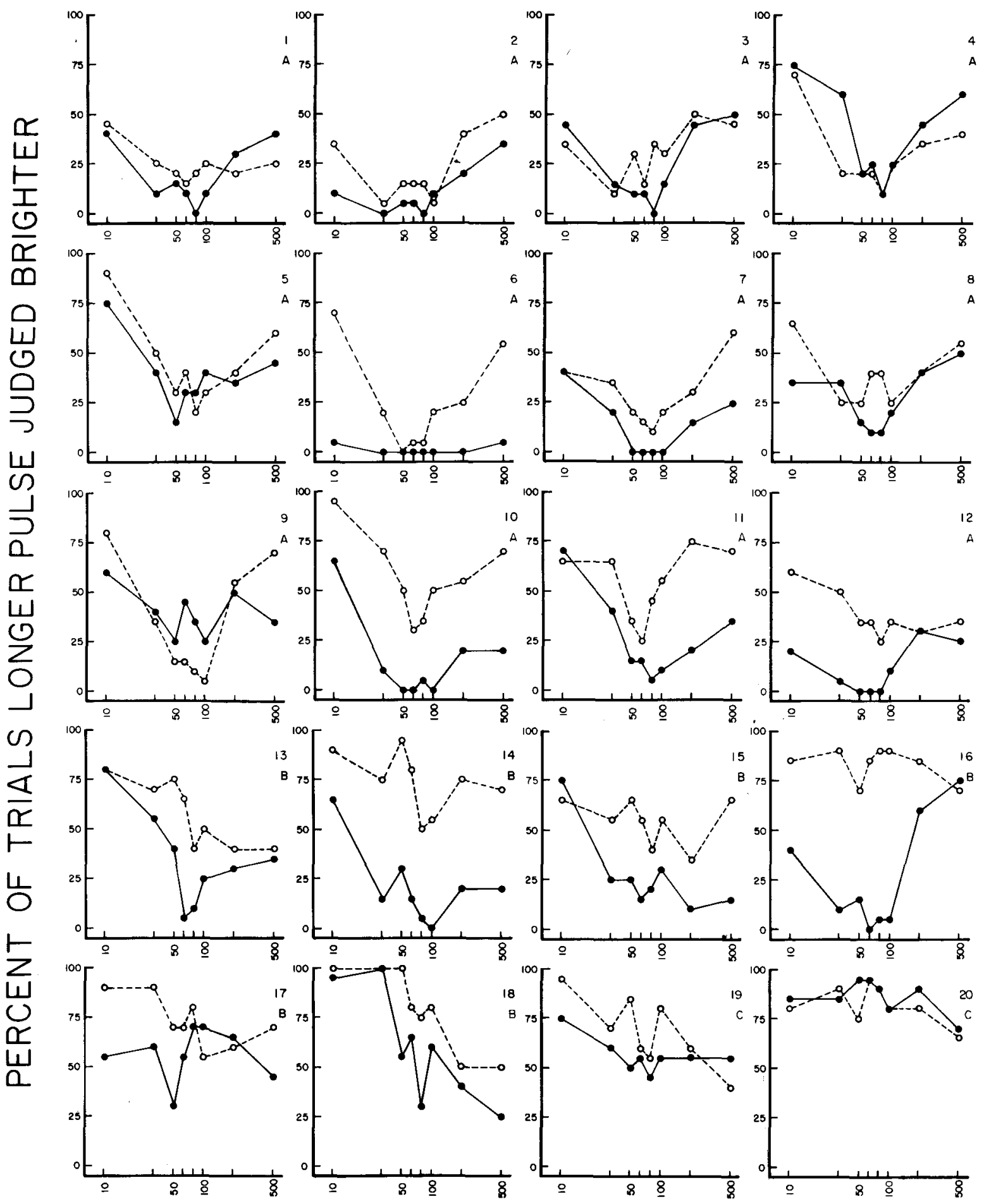

\section{DURATION OF SHORTER PULSE (MSEC)}

Figure 3. Results for Condition 1 (see text). 
GENERAL INSTRUC./RANDOM SEQ.
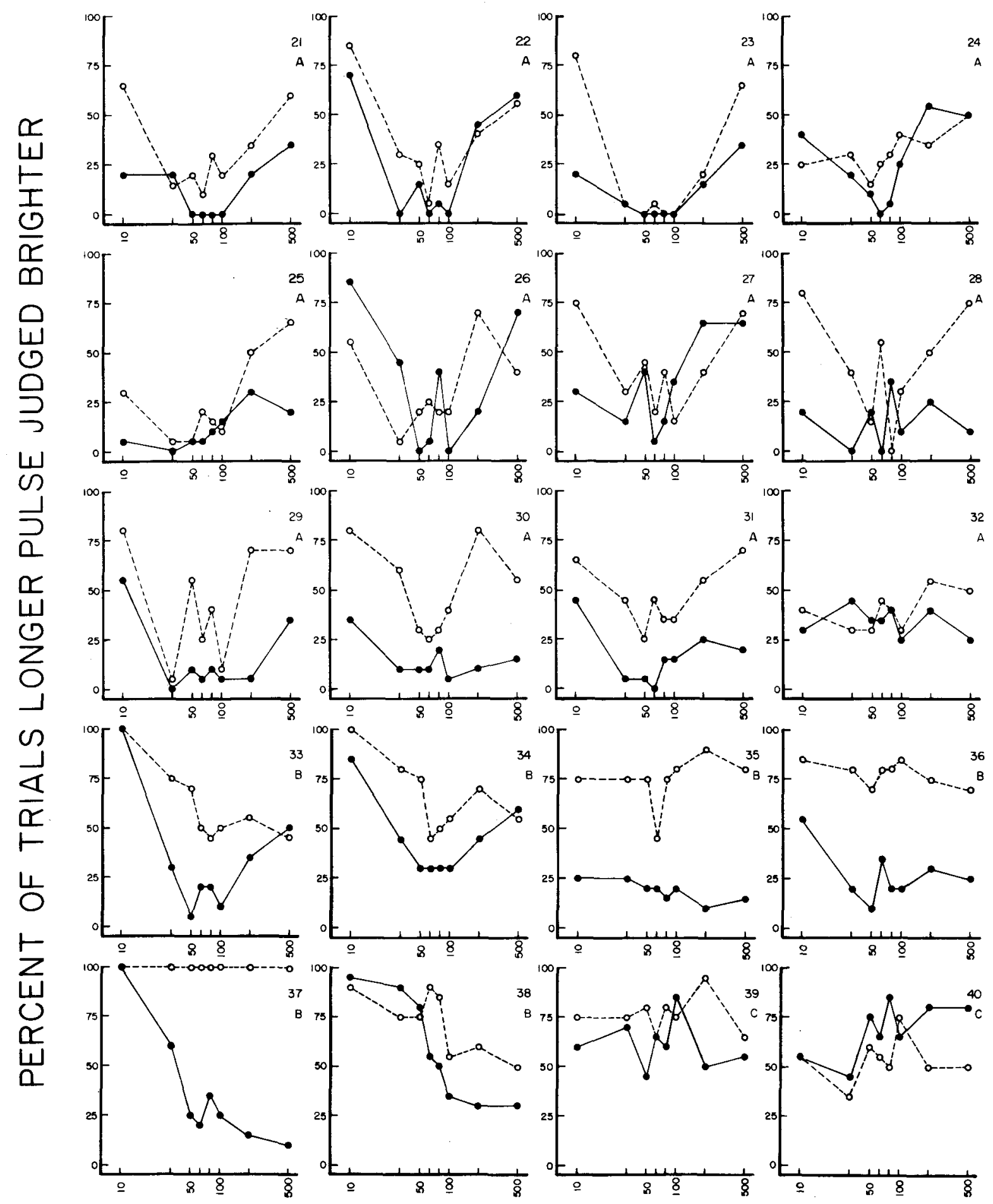

DURATION OF SHORTER PULSE (MSEC)

Figure 4. Results for Condition 2. 
SPECIFIC INSTRUC./ORDERED SEQ.

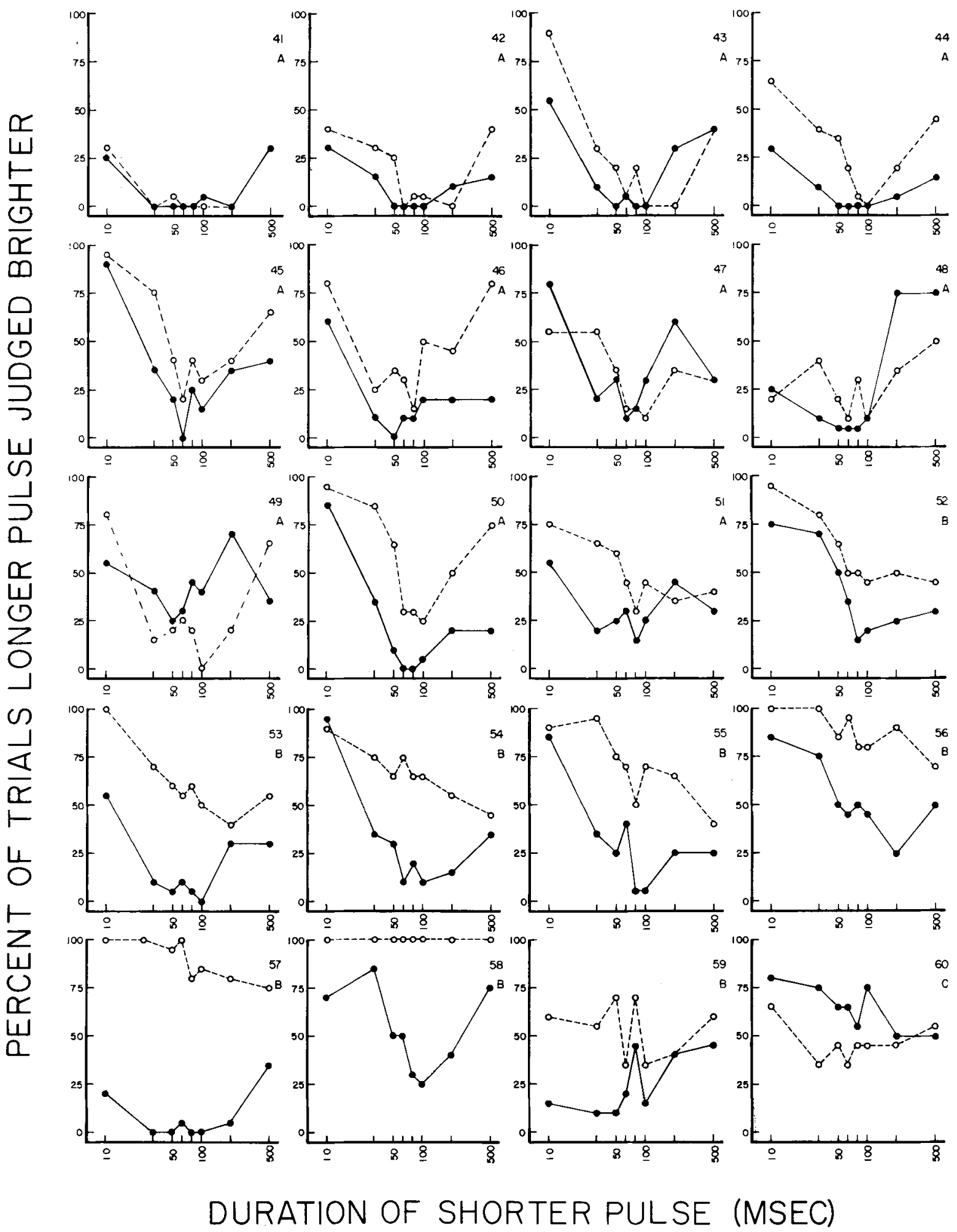

Figure 5. Results for Condition 3. 
SPECIFIC INSTRUC./RANDOM SEQ.
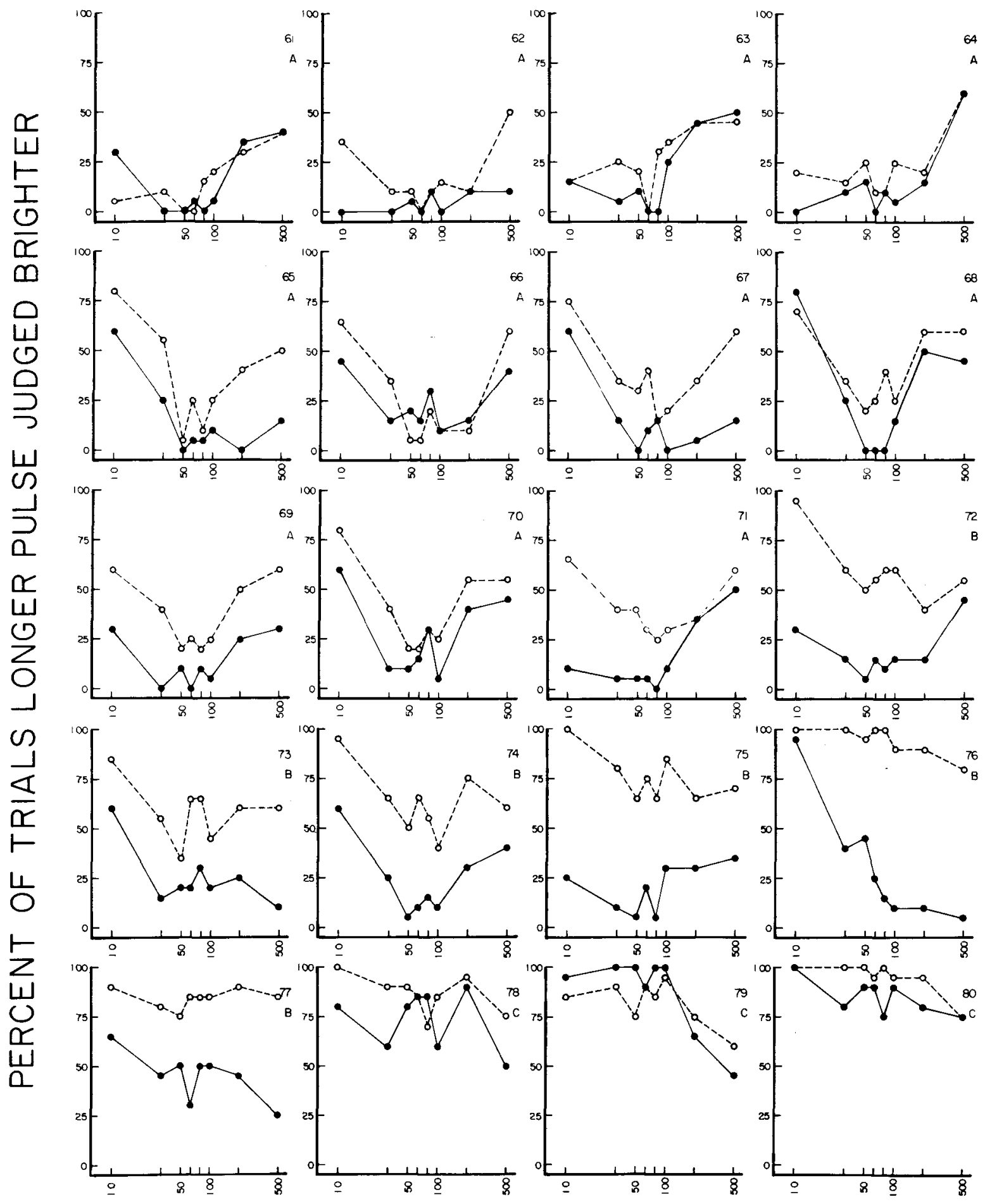

DURATION OF SHORTER PULSE (MSEC)

Figure 6. Results for Condition 4. 
tion of simultaneous onset is often elevated relative to that for simultaneous offset, but significant brightness enhancement effects are always present for both asynchrony conditions and the asynchrony functions are frequently approximately parallel. Most Type A observers show minimum values (peak brightness) in a range from 50 to $100 \mathrm{msec} ; 57.5 \%$ of all observers are Type $A$.

Type B observers show a significant brightness enhancement effect for the simultaneous offset condition, but not for the simultaneous onset condition. This result is similar to that obtained by Raab and Osman (1962) and occurred in $32.5 \%$ of all observers.

Type C observers show no Broca-Sulzer effect under either simultaneous onset or offset conditions. The data for Type $\mathrm{C}$ observers generally are at a level above $50 \%$ for shorter pulses and asymptotic at a level near $50 \%$ for longer pulses. Ten percent of the observers are Type $C$.

Table 1 gives the number of Type A, Type B, and Type $C$ observers for the four experimental conditions. This table and Figures 3-6 suggest that there are no statistical differences in the distribution of the three classes of observers for the four principle experimental conditions. Neither instructional set nor sequencing of pulse presentations had any significant influence on the relative frequency of occurrence of the three classes of observers.

In order to confirm this observation, the data were subjected to a four-way, repeated-measures analysis of variance (ANOVA), with between-subject factors of instructional set (two levels) and sequence of stimulus presentation (two levels) and within-subject factors of temporal asynchrony (two levels) and shorter pulse duration (eight levels). The ANOVA indicated significant effects of asynchrony (simultaneous onset vs. offset) $[F(1,76)=91.05, p<<$
Table 1

Distribution of Type A, B, and C Observers Across Experimental Conditions

Sequence

\begin{tabular}{cccccc} 
& \multicolumn{4}{c}{ Sequence } \\
\cline { 2 - 5 } Type & \multicolumn{2}{c}{ General Instructions } & \multicolumn{2}{c}{ Specific Instructions } \\
\cline { 2 - 5 } \cline { 5 - 6 } & Ordered & Random & Ordered & Random & Total \\
\hline A & 12 & 12 & 11 & 11 & 46 \\
B & 6 & 6 & 8 & 6 & 26 \\
C & 2 & 2 & 1 & 3 & 8 \\
\hline
\end{tabular}

$.01]$ and shorter pulse duration $[F(7,532)=67.56$, $\mathrm{p}<<.01]$. On the other hand, neither the effect of instructional set $[F(1,76)=.010]$ nor the effect of stimulus sequence $[F(1,76)=.051]$ reached statistical significance. Thus, although there exist at least three types of observers, their occurrence is not readily influenced by general vs. specific instructions or by ordered vs. random sequencing of stimuli. $^{2}$

The data of Figures 3-6 exhibit enough variability among individuals of a given type to raise the possibility that our binomial criterion for categorizing types may not have been a sufficient one. Accordingly, in Figure 7, we plotted the average data for each type of observer collapsed across our original experimental conditions. Error bars indicate the $95 \%$ confidence interval for the means. This type of plot is justified by the lack of significant main effects and interactions involved between group variables in the ANOVA.

The functions in Figure 7 are in complete accord with the generalizations made concerning the data of individual observers. Type A observers, as a group, show statistically significant enhancement effects for both asynchrony conditions (as before, filled circles/

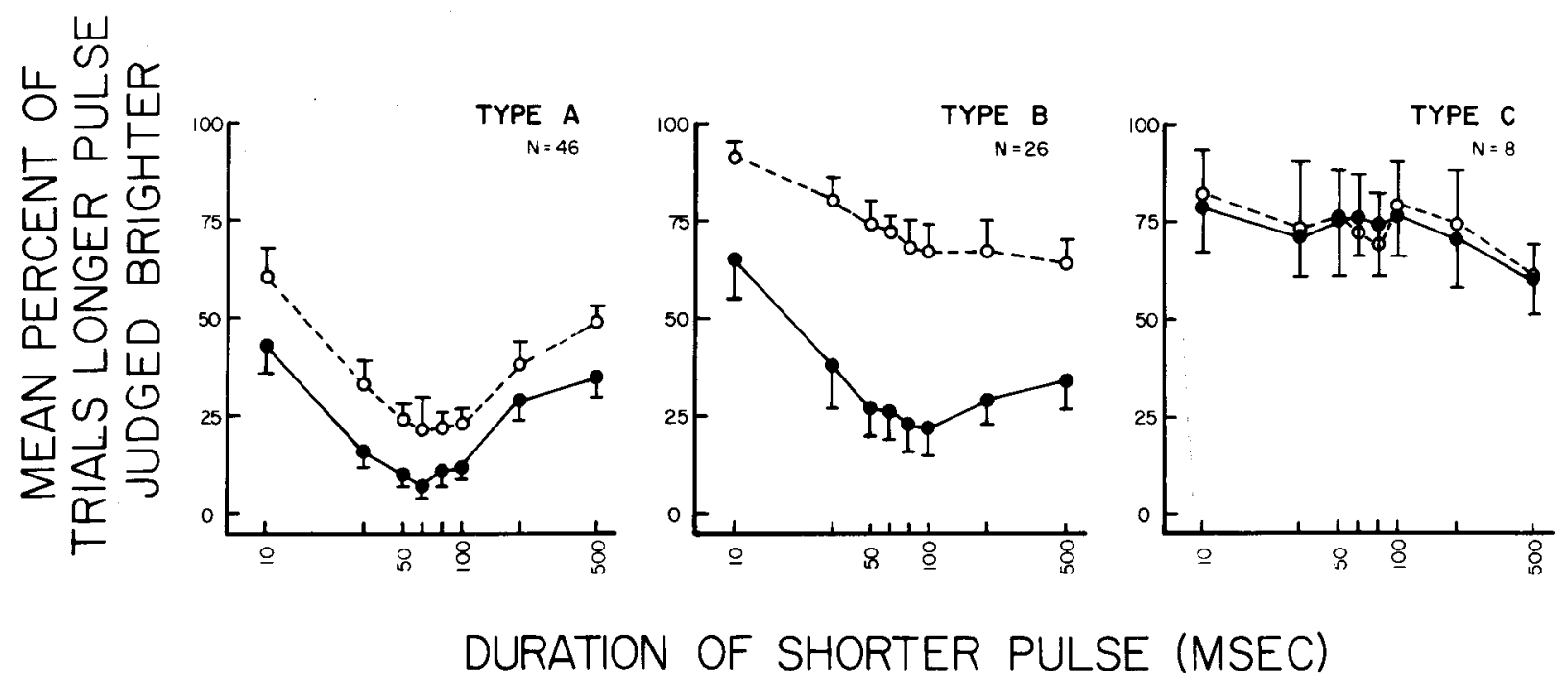

Figure 7. Average data for the three classes of observers collapsed across experimental conditions. Error bars indicates $95 \%$ confidence intervals. 
solid lines indicate simultaneous offset; open circles/ broken lines are for the simultaneous onset condition). The tendency noted above for the simultaneous offset condition to produce a lower overall function is evident in the mean data. Type B observers, as a group, show a lack of effect for simultaneous onset and a significant effect for simultaneous of fset comparable to that for Type A observers. For Type C observers, there is no statistical difference between asynchrony conditions, and for both conditions there is a lack of brightness enhancement. The data of Figure 7 establish statistically significant differences between and within types of observers that are consistent with the description of the individual data offered above.

A possible confounding artifact in our paradigm would be a position preference (left or right) in making the brightness judgment. Accordingly, we conducted a chi-square test of association comparing the number of times the longer pulse was judged brighter for the left vs. the right presentation positions across all short pulse durations for each of the asynchrony conditions. Among the obtained chisquare values, only three cases were significant at the $95 \%$ level or greater. Since, with a large sample of chi-square values, one would expect by chance no more than $5 \%$ of the values to reach statistical significance, we conclude that no position preference is evident in our data.

\section{DISCUSSION}

We have shown that, for a paradigm involving simultaneous brightness comparisons between two light pulses, three behavioral classes of psychophysical observers may be identified. Furthermore, the instructional sets and stimulus sequences we employed had no influence on the distribution of Type $A$, $B$, and $C$ observers. Why do these different classes of observers exist? We note, first of all, that the present study was intended to identify different classes of observers, but was not designed to analyze how types of observers are differentiated. Thus, a number of explanations are possible. We would like to explore two possibilities: One is that the three classes of observers possess different visual systems, that is, that the underlying anatomical and/or neurophysiological mechanisms or responses responsible for brightness perception differ among the classes. The second hypothesis is that observers adopt qualitatively or quantitatively different perceptual criteria in making the brightness judgment.

The idea that the three classes of observers possess different basic visual processing mechanisms can be related to the recent postulate that the occurrence of the Broca-Sulzer effect is dependent upon activation of a "transient" or "phasic" visual process- ing mechanism (Bowen \& Nissen, 1979; Kitterle \& Corwin, 1979). ${ }^{3}$ We might assume, for example, that Type $\mathrm{C}$ observers have a weak transient processing system, and hence show no enhancement effects. If a weakness in the transient processing mechanism is possible, it might manifest itself in tests of visual function other than the Broca-Sulzer phenomenon, for example, flicker detection, absolute thresholds, etc. Since we tested only Snellen acuity for our observers, this issue requires further study.

While this sort of hypothesis might account for the existence of Type $\mathrm{C}$ and Type A observers, it may not be able to explain the occurrence of the Type B observers, who show brightness enchancement for conditions of simultaneous offset but not for simultaneous onset. This result cannot be due to a weak transient processing mechanism, since the Broca-Sulzer phenomenon can be seen by such observers. ${ }^{4}$

Another possible neurophysiological difference among observers is suggested by the "color coding" theory of brightness enhancement (Wasserman, 1966). This model of the Broca-Sulzer effect attributes brightness enhancement to the action of chromatic opponent-color mechanisms and would suggest, for example, that Type $\mathrm{C}$ observers may have defective color vision, as do about $10 \%$ of the general population (Hsia \& Graham, 1965). We did not screen our observers for color vision in this experiment, but in a subsequent study (Bowen, Sekuler, Markell, \& Owlsley, Note 1) we have uncovered four new Type C observers, all of whom have normal color vision. Therefore, the "color coding" model does not apply. Furthermore, the model would encounter the same difficulties accounting for Type B observers as are discussed above. In general, it does not seem likely that the presence or absence of specific neurophysiological mechanisms will account for our data, but we present below a model in which variation in the magnitude of some neurophysiological response among observers might explain the existence of different types of observers.

An alternative approach is to consider that the different classes of observers adopt different perceptual criteria for assessing pulse brightness. It is difficult, however, to discuss this issue definitively, because we have no prior knowledge of how the sensory response to a pulse of light varies as a function of time. A variety of possible criteria applied to a number of possible temporal "features" of the sensory response could account for the data. Classes of observers might be differentiated on the basis of whether they assess the brightness of a pulse of light with respect to peak sensory activity, or as a temporal integral of sensory activity, or with respect to any sustained level of activity in the sensory response, and so on. The anticipated effects of onset vs. offset pulse asynchrony will likewise depend on 
assumptions concerning the underlying sensory response. Because any number of assumptions about the nature of the sensory response to a pulse of light can be put forth, a critical discussion of perceptual criteria for judging brightness is thereby limited.

On the other hand, if we accept criterion differences as a possible candidate for understanding the data, it should not be surprising that different observers may adopt criteria differentially: Bernstein and his co-workers (Bernstein, Proctor, Belcher, \& Schurman, 1974; Bernstein, Proctor, Proctor, \& Schurman, 1973) have demonstrated that in a metacontrast masking paradigm, a given observer may shift his criteria for judging the brightness of a masked stimulus from a comparative judgment (in which the masking stimulus provides a frame of reference) to an absolute judgment as the delay between test and subsequent masking stimulus is increased. In our own paradigm, potential differences in criteria are strongly related to the temporal asynchrony conditions; it is interesting to note that we have not observed the converse of Type B, that is, an individual who shows the Broca-Sulzer effect for simultaneous onset but not simultaneous offset. For many observers, brightness comparisons involving offset simultaneity produce the most robust brightness enhancement effects. It would be valuable to establish in future work whether examples of occurrence and nonoccurrence of the Broca-Sulzer effect can be obtained with a single-stimulus method of measurement, for example, a scaling technique such as magnitude estimation (Raab, 1962).

If our observers did adopt differing perceptual criteria, it is somewhat surprising that these were not affected by our experimental conditions and that they apparently are not readily manipulated. It would be of interest to establish whether many sessions of practice with the judgment could alter the distribution of observers.

Thus far, we have discussed the possible role of neurophysiology and perceptual criterion in determining our results in terms of relatively specific cases, that is, presence vs. absence of neurophysiological mechanisms, adoption of particular criteria, etc. We have also analyzed the data as representing evidence for discrete and independent classes of observers. We can be somewhat more abstract in discussing the possible basis of our effects, and we can demonstrate that the occurrence of behaviorally distinct observer types can also be accounted for by continuous distributions within the population of either neurophysiological responses or criterion level. Consider the following model of our data based on this proposition. ${ }^{5}$ The model will apply with either of two assumptions: one assumption is that all individuals have identical neurophysiological responses to pulses of light but differ in their perceptual criterion or decision level according to a normal distribution of such criteria. Here we also assume that a given individual is more likely to manifest the Broca-Sulzer phenomenon for simultaneous offset conditions than for simultaneous onset, and that his criteria for these conditions differ by a fixed amount, with the result that $t w o$ overlapping normal distributions of criteria, one for each of the two asynchrony conditions, are generated.

The alternative assumption is that individuals differ in the size of the neurophysiological response to a pulse of light according to a normal distribution, but have a fixed and equal criterion or decision level for manifesting brightness enhancement. The additional assumption in this case is that the size of the neurophysiological response is greater by a fixed amount for simultaneous offset than for simultaneous onset conditions for a given individual, so that, again, two overlapping normal distributions of response are generated.

These alternative sides to the model are illustrated schematically in Figure 8. To generate the occurrence of Type A, B, and C observers in the behavioral domain, we assume the existence of a decision point along the abscissa of the figure (indicated by the vertical line), above which brightness enhancement will be seen and below which it will not. The normal distributions shown and the decision point illustrated have been positioned so as to generate the empirical proportions of Type A, B, and C observers evident in our data. For example, the offset distribution is positioned so that $10 \%$ of the cases in this distribution fall below the decision point. These are Type C observers. The $42.5 \%$ of the cases from the onset distribution below the decision point are Type B and Type C observers $(32.5 \% \mathrm{~B}, 10 \% \mathrm{C})$. Type A observers comprise the $57.5 \%$ of cases from the onset distribution above the decision point. Finally, the $90 \%$ of the offset distribution above the decision point represents the $57.5 \%$ Type $A$ and $32.5 \%$ Type B observers.

This analysis illustrates that the population is not necessarily comprised of independent classes of observers. The analysis can be applied equally well for the assumption of identical criterion or for identical neurophysiology among observers. It also has potential as a point of departure for investigating the basis of our effects, perhaps in terms of devising ways of studying the hypothetical distributions making up the model. If this general model has merit, it may turn out that variations in both criterion and neurophysiology contribute to creating the three classes of observers. As far as our results are concerned, even though the model is plausible, the shorthand designation of Type $\mathrm{A}, \mathrm{B}$, and $\mathrm{C}$ observers from a behavioral standpoint seems appropriate at this point.

We have demonstrated significant individual differences in the relation between brightness and pulse duration. We now suggest that potential experimen- 


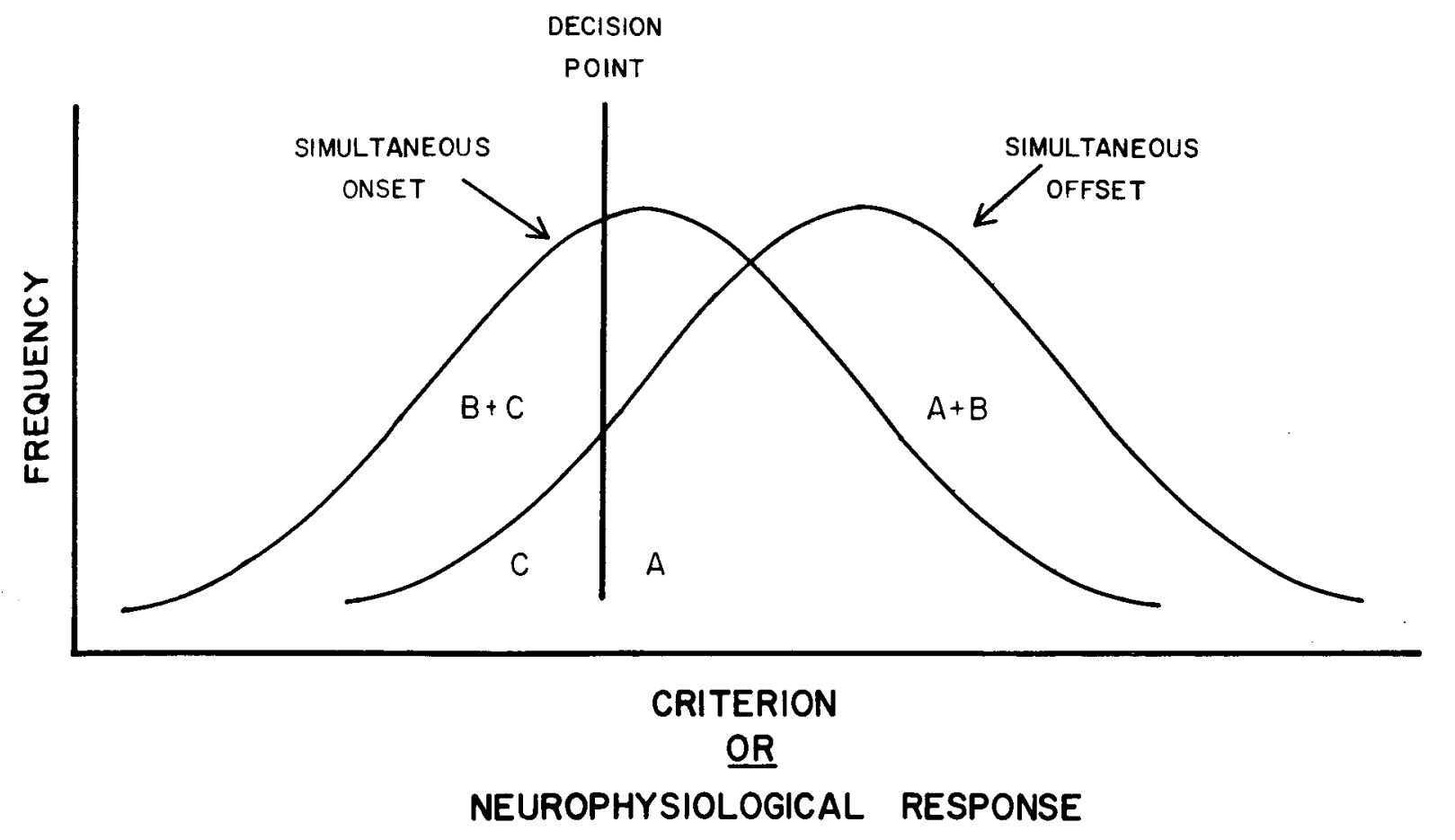

Figure 8. Schematic representation of a model for explaining the existence of three classes of observers (see text).

tal subjects for studies of temporal brightness enhancement be prescreened to establish to which class of observers they belong. Otherwise, erroneous conclusions might be drawn to the effect that given stimulus conditions do or do not produce brightness enhancement, when, in fact, obtained results could be due to differential sampling of A-, B-, or C-type observers. Finally, the present results show clearly that the effects of pulse asynchrony observed by Raab and Osman (1962) are not general and apply only to some observers. Our results indicate that pulse asynchrony should properly be included as a principal variable in any study of the Broca-Sulzer effect which involves simultaneous brightness discrimination.

\section{REFERENCE NOTE}

1. Bowen, R. W., Sekuler, R., Markell, K. A., \& Owsley, C. Individual differences in pulse brightness perception: Tests of visual function. Manuscript in preparation.

\section{REFERENCES}

AlPE RN, M. Simultaneous brightness contrast for flashes of light of different durations. Investigative Ophthalmology, 1963, 2, 47-54.

Bernstein, I. H., Proctor, R. W., Belcher, J., \& Schurman, D. L. An analysis of U-shaped metacontrast. Perception \& Psychophysics, 1974, 16, 329-336.

Bernstein, I. H., Proctor, J. D., Proctor, R. W., \& Schurman, D. L. Metacontrast and brightness discrimination. Perception \& Psychophysics, 1973, 14, 293-297.
Boynton, R. M. Some temporal factors in vision. In W. A. Rosenblith (Ed.), Sensory communication. New York: Wiley, 1961.

Bowen, R. W., \& Nissen, M. J. Luminance, not brightness, determines temporal brightness enchancement with chromatic stimuli. Journal of the Optical Society of America, 1979, 69, 581-584.

Bowen, R. W., \& Pokonny, J. Target edge sharpness and temporal brightness enchancement. Vision Research, 1978, 18, 1691-1695.

Broca, D., \& Sulzer, A. La sensation lumineuse en fonction du temps. Journal de Physiologie et de Pathologic Generale, $1902,4,632-640$.

Enroth-Cugetl, C., \& Robson, J. G. The contrast sensitivity of retinal ganglion cells of the cat. Journal of Physiology (London), 1966, 187, 517-522.

Gordon, J., \& Hoop, D. C. Anatomy and physiology of the frog retina. In K. V. Fite (Ed.), The amphibian visual system: A multidisciplinary approach. New York: Academic Press, 1976.

Gouras, P. Identification of cone mechanisms in monkey ganglion cells. Journal of Physiology (London), 1968, 199, 533-547.

Hsia, Y., \& Graham, C. H. Color blindness. In C. H. Graham (Ed.), Vision and visual perception. New York: Wiley, 1965.

INGLING, C., \& Drum, B. Retinal receptive fields: Correlations between psychophysics and electrophysiology. Vision Research, $1973,13,1151-1163$.

KATz, M. Brief flash brightness. Vision Research, 1964, 4, 361 373.

Kitterle, F. L., \& Conwin, T. R. Enchancement of apparent contrast in flashed sinusoidal gratings. Vision Research, 1979, 19, 33-40.

MacLeon, D. I. A. Visual sensitivity. Annual Review of Psychology, 1978, 29, 613-645.

RAAB, D. Magnitude estimation of the brightness of brief foveal stimuli. Science, 1962, 135, 42-44. 
RAAB, D., \& Osman, R. Effect of temporal overlap on brightness matching of adjacent flashes. Journal of the Optical Society of America, 1962, 52, 1174-1178.

Schille R, P. H., \& MAlpeli, J. G. Properties and tectal projections of monkey retinal ganglion cells. Journal of Neurophysiology, 1977, 40, 428-445.

WASSERMAN, G. S. Brightness enhancement and opponent-colors theory. Vision Research, 1966, 6, 689-699.

\section{NOTES}

1. As far as we can determine, the figure of 80 observers is not far from the total number of observers who have participated in studies of the Broca-Sulzer effect since 1902.

2. Within the ANOVA, one second-order interaction (Sequence by Duration) and one third-order interaction (Sequence by Asynchrony by Duration) barely attained statistical significance $[F(7$, $532)=2.84$ and $F(7,532)=2.24$, respectively]. We have no explanation for such interactions.

3. Many recent experimental developments in the study of spatiotemporal vision point to the conclusion that the human visual system contains at least two parallel processing channels, called "transient" and "sustained," which have different spatial and temporal response properties (see MacLeod, 1978, for a recent review). The transient mechanism is thought to be most sensitive to rapid fluctuations in luminance (higher temporal frequencies) or rapid image motion, but is relatively insensitive to fine spatial detail (high spatial frequencies). The sustained mechanism responds best to low temporal frequencies and high spatial frequencies. These psychophysical properties of sustained and transient mechanisms have been shown to be correlated with the response properties of " $\mathrm{X}$ " and " $\mathrm{Y}$ " cells in the visual system of the cat (Enroth-Cugell \& Robson, 1966) and "tonic" and "phasic" cells in the monkey visual system (Gouras, 1968; Schiller \& Malpeli, 1977). (See Ingling \& Drum, 1973, for an expository discussion.)

4. Raab and Osman (1962) hypothesized that Type B effects were due to mutual inhibitory interactions between the pulses to be compared. Our conditions of stimulation should have minimized the possibility for interactions. Moreover, hypotheses of this type will not account for Type A and Type C observers.

5. We are grateful to D. C. Hood for suggesting this general analysis. A similar approach to analyzing classes of feature detectors exists in the neurophysiological literature (Gordon \& Hood, 1976).

(Received for publication September 13, 1979; revision accepted February 22, 1980.) 